\title{
TRABAJO COLABORATIVO DOCENTE Y SU IMPACTO EN LA GESTIÓN PEDAGÓGICA
}

\section{COLLABORATIVE TEACHING WORK AND ITS IMPACT ON PEDAGOGICAL MANAGEMENT}

\section{Autor: Angel Marcelo Cueva Bravo}

ORCID ID: https://orcid.org/0000-0002-6505-1672

E-mail de contacto: angelocueva@ hotmail.com

Articulo recibido: 31 de Marzo del 2019.

Articulo revisado: 28 de Agosto del 2019

Articulo aprobado: 6 de Diciembre del 2019

Licenciado en Ciencias de la Educación mención Educación Básica egresado de la Universidad Nacional de Loja (Ecuador) con 10 años de experiencias en la docencia, es Rector del Centro Educativo Comunitario Intercultural Bilingüe "Juan Pinchu". Maestrante en la Universidad Nacional Mayor de San Marcos de Lima (Perú), mención Gestión de la Educación.

\section{Resumen}

Los deficientes resultados en la gestión pedagógica en el ámbito escolar, ha llevado a la necesidad de transformar la enseñanza. En la actualidad, la reflexión y el trabajo colaborativo con colegas se conciben como claves en el proceso de gestión pedagógica. A partir de la experiencia de un grupo de docentes de la escuela de Educación Básica "José Ingenieros" Cantón Loja, determinando la contribución que tiene el trabajo colaborativo docente y su impacto en la gestión pedagógica. Destacando aspectos como el reconocimiento de debilidades y fortalezas, el escuchar y aprender de la experiencia de otros y la gestión pedagógica concreta. Por otra parte, estas reflexiones contribuyen a la transformación en diferentes niveles: del profesor y de la comunidad escolar. El hecho de que sea un grupo diverso, que incluye a profesores del sistema escolar, genera reflexiones enriquecedoras, que ayudan a transformar la gestión pedagógica.

Palabras clave: gestión pedagógica, reflexión, trabajo colaborativo, educación.

\section{Abstract}

The poor results in pedagogical management in the school environment, has led to the need to transform teaching. Currently, reflection and collaborative work with colleagues are conceived as keys in the process of pedagogical management. Based on the experience of a group of teachers from the Basic Education School "José Ingenieros" Cantón Loja, determining the contribution of collaborative teaching work and its impact on pedagogical management. Highlighting aspects such as the recognition of weaknesses and strengths, listening and learning from the experience of others and concrete pedagogical management. On the other hand, these reflections contribute to the transformation at different levels: of the teacher and of the school community. The fact that it is a diverse group, which includes teachers of the school system, generates enriching reflections that help transform pedagogical management.

Keywords: pedagogical management, reflection, collaborative work, education.

\section{Sumário}

Os fracos resultados na gestão pedagógica no ambiente escolar levaram à necessidade de transformar o ensino. Atualmente, a reflexão e o trabalho colaborativo com os colegas são concebidos como chaves no processo de gestão pedagógica. Com base na experiência de um grupo de professores da Escola de Educação Básica "Cantina José Ingenieros", determinando a contribuição do trabalho colaborativo de ensino e seu impacto na gestão pedagógica. Destacar aspectos como o reconhecimento de pontos fracos e fortes, ouvir e aprender com a experiência de outras pessoas e uma gestão pedagógica concreta. Por outro lado, essas reflexões contribuem para a transformação em diferentes níveis: o professor e a comunidade escolar. O fato de ser um grupo diverso, que inclui professores do sistema escolar, gera reflexões enriquecedoras, que ajudam a transformar a gestão pedagógica.

Palavras-chave: gestão pedagógica, reflexão, trabalho colaborativo, educação. 


\section{Ciencia y Educación (ISSN 2707-3378) \\ Vol. 1 No. 1 \\ Enero del 2020}

\section{Introducción}

A nivel mundial, y hace ya algunas décadas, se viene hablando de una «crisis en la educación» (Pozo y Gómez, 1998). Esta se expresa no solo en una incomprensión por parte de los docentes de un conjunto de conceptos pedagógicos básicos, sino también en carencias en el desarrollo de capacidades y en la comprensión del trabajo colaborativo como actividad humana.

En Ecuador, los resultados de pruebas como PISA o TIMSS, muestran que la situación es especialmente preocupante y transversal a nivel educativo. Siendo reconocido el rol fundamental del docente en el aprendizaje de los alumnos (Barber y Mourshed, 2008; Cofré, et al., 2010), una de las grandes causas de esta crisis sería la predominancia de una enseñanza frontal $\mathrm{y}$ descontextualizada, que no considera ni los intereses de los alumnos ni sus conocimientos previos.

Lo anterior, además de afectar el desarrollo de actitudes, conocimiento y habilidades, ha llevado a los docentes a una falta de motivación por enseñar. A partir de lo anterior, surge la urgente necesidad de reformar la educación, y en particular transformar el uso de la gestión pedagógica de los docentes dentro de las unidades educativas. Pero es necesario conocer bajo qué condiciones un docente transforma su gestión pedagógica.

Históricamente, no solamente en Ecuador, el diseño e implementación de nuevas reformas educacionales ha estado a cargo de académicos y encargados de la política pública, con poca participación de los docentes en el proceso (Lang, 2007). Si bien ha habido diversas iniciativas de desarrollo profesional docente orientadas a transformar la gestión pedagógica, éstas se han llevado a cabo mayoritariamente desde una perspectiva vertical («de arriba abajo», Muñoz y Vanni, 2008), distinguiendo a un ministerio central de carácter técnico que diseña, un ministerio regional/provincial que ejecuta, y una escuela o liceo que recibe la intervención, sin mayor incidencia en el proceso.

Por su parte, las instancias de desarrollo profesional docente suelen darse también en un formato vertical, donde el «experto» de la universidad instruye al docente en cómo debe actuar, sin considerar su experiencia y contexto, asumiendo este último el rol de técnico que va a ejecutar una tarea. En contraste, y desde esta postura, los docentes que se enfrentan a una instancia de desarrollo profesional formal deben ser tratados en su rol profesional (no técnico), esto es, deben considerar que el profesor es creativo y capaz de tomar decisiones según su capacidad de detectar necesidades y responder a ellas con conocimientos, los cuales se han construido tanto en procesos de formación como a partir de su propia experiencia docente.

\section{Desarrollo}

En relación con el desarrollo profesional de los docentes en particular, Bell y Gilbert proponen en 1996 un modelo basado en un estudio que investiga la manera en que un conjunto de docentes cambia su gestión pedagógica desde un enfoque transmisivo hacia un enfoque constructivista de enseñanza (Simón \& Campbell, 2012). El modelo propone tres ámbitos de desarrollo: el ámbito personal (involucra sentimientos, motivación, disponibilidad y compromiso del profesor), el profesional (conocimientos y concepciones, así como visualización de los efectos de la práctica en los estudiantes) y el social (valoración del trabajo colaborativo y el contexto de trabajo) (Bell, 1998; Mellado, 2001).

Estos ámbitos estarían relacionados entre sí, y se desarrollan de manera paralela y continua, gatillados por procesos de reflexión y trabajo 


\section{Ciencia y Educación (ISSN 2707-3378) \\ Vol. 1 No. 1 \\ Enero del 2020}

colaborativo. Desde esta perspectiva, y más allá del ámbito del desarrollo profesional de los docentes, existen diversas investigaciones donde el trabajo colaborativo docente aparece como un instrumento promotor del cambio (Ávalos, 2011).

Un estudio de Schnellert et al. (como se citó en Ávalos, 2011), señala que la gestión pedagógica docentes, más que estar relacionados con los años de experiencia, estarían gatillados por el grado de involucramiento de los docentes en una indagación reflexiva y colaborativa. Así, el trabajo colaborativo docente pareciera ser el factor clave en la transformación de la gestión pedagógica: un docente que no problematiza su práctica y no reflexiona sobre ella no sentirá la necesidad de actuar de manera diferente en el aula. Por otra parte, el acto de reflexionar sobre el trabajo colaborativo se potencia especialmente en el contexto de las comunidades de aprendizaje.

La retroalimentación y el acceso a nuevas ideas e información se posibilitan a través de la interacción y el diálogo con otras personas en el marco del trabajo colaborativo, que a su vez contribuye para crear una cultura de aprendizaje y una comunidad, donde el aprendizaje es valorado y estimulado (Chou, 2011). El trabajo docente en torno a estas comunidades de aprendizaje además incidiría positivamente en el aprendizaje de los estudiantes de los docentes participantes de estas comunidades (Vescio, Ross, \& Adam, 2008).

Finalmente, la Investigación Acción Colaborativa (IAC) se presta como un escenario interesante para potenciar la reflexión a través de la colaboración. La IAC se entiende como la investigación que los profesores hacen con el propósito de comprender y mejorar su propia gestión pedagógica profesional. Se le considera una forma efectiva de desarrollo profesional docente, especialmente cuando se realiza de manera colaborativa (Chou, 2010; Lebak \& Tinsley, 2010; Ponte, 2010).

El presente trabajo se enmarca en una propuesta de desarrollo profesional, concretada en la realización de una investigación con enfoque cualitativo realizada con la población que estuvo constituido por los docentes de la escuela de educación básica José Ingenieros. Este trabajo tiene como objetivos: (a) comprender la manera en que el trabajo colaborativo contribuye a los procesos de gestión pedagógica orientada a la transformación de la enseñanza por parte de los docentes y (b) analizar la incidencia del trabajo colaborativo docente en la planificación del aprendizaje

El trabajo colaborativo involucra activamente a los profesores en la gestión pedagógica, valida a los educadores como productores de conocimiento y reconoce su rol en el desarrollo profesional y la toma de decisiones (Burbank \& Kauchak, 2003, p. 499), lo que en esta experiencia contribuyó a los procesos de transformación de la gestión pedagógica de los profesores.

Tal como indica Vescio, Ross y Adam (2008), a través de la indagación colaborativa, los docentes exploran nuevas ideas y revisan se accionar en la gestión pedagógica actuales y evidencias del aprendizaje de los estudiantes, usando procesos que los respetan como expertos en determinar qué es lo necesario para mejorar la propia práctica y mejorar el aprendizaje de los estudiantes, animando así a los profesores a vincular conocimientos profesionales, académicos y de su conocimiento personal docente y de sus colegas (Ponte, 2010).

La investigación colaborativa supone un proceso formalizado y sistemático de reflexión sobre la propia práctica de los docentes, quienes son capaces de estructurar conscientemente situaciones y problemas, lo que según Korthagen 


\section{Ciencia y Educación (ISSN 2707-3378) \\ Vol. 1 No. 1 \\ Enero del 2020}

y Wubbels (2001) es parte del pensamiento docente que se distingue como reflexivo. El conjugar los eventos presentes con una postura reflexiva lleva a los docentes a cambiar las bases de su pensamiento, desde la anécdota hacia el pensamiento profesional crítico, desde la acción rutinaria o habitual - muchas veces intuitivahacia una acción basada en una autoevaluación y una mayor conciencia social, cultural y política, así como una mayor flexibilidad y creatividad (Mitchell, Reilly, \& Logue, 2009).

En este mismo aspecto, durante el proceso de investigación-acción colaborativa, se puede observar y constatar un desarrollo de conocimientos sobre el contexto educativo que pasan a ser relevantes para la comprensión y toma de conciencia del proceso de enseñanza del contenido, evidenciado en los distintos momentos de reconocimiento de las distintas realidades en escuelas. Del mismo modo, se aprecia una fuerte influencia de lo que podríamos llamar aspectos afectivos, como las creencias personales sobre autoeficacia logradas o desarrolladas durante el proceso colaborativo (Garritz, 2010; Park \& Oliver, 2008).

Como consecuencia, los docentes comienzan a transformar su gestión pedagógica hacia unas más centradas en los estudiantes, aumentando la colaboración, el empoderamiento y el aprendizaje continuo (Vescio et al., 2008), contribuyendo a la construcción del conocimiento del profesorado y a su autoconfianza (Chou, 2010). En palabras de Bell (1998, p. 683): «La reconstrucción de nuestro conocimiento colectivo de lo que significa ser docente», como primer paso para que ocurra la innovación de la gestión pedagógica (Bell, 1998).

\section{Conclusión}

Por último, uno de los aspectos más interesantes del proyecto es que la indagación colaborativa permitió establecer un puente entre teoría y práctica, porque promueve el uso del conocimiento tácito de los profesores para que colaborativamente identifiquen e implementen estrategias para hacer frente a las cuestiones pedagógicas, que, cuando se combinan con el conocimiento formal (como el que se encuentra en la literatura), fomentan la mejora de la enseñanza (Albers, 2008).

Para aquellos docentes del grupo que trabajamos en la formación inicial/continua de profesores e investigamos la naturaleza del desarrollo profesional de docentes, la participación en el grupo ofreció una oportunidad especial de hacer esta vinculación teoría-práctica. Si bien existen numerosos estudios acerca de la naturaleza de las creencias docentes, su conocimiento y su gestión pedagógica orientados a comprender los factores que ayudan a mejorar la calidad de la enseñanza, estos son en su gran mayoría realizados por investigadores externos, donde los docentes participantes no han sido necesariamente los promotores de la investigación (Loughran, 2007).

Esto cambia cuando son los propios docentes los que investigan la gestión pedagógica en contextos de diversidad. El hecho de ser nosotros mismos objetos y sujetos de investigación y compartir en un mismo nivel los problemas en la gestión pedagógica permitió tener una mirada más amplia de lo que supone el proceso de formación docente, incluyendo el proceso de aprender a enseñar.

Llegará el día en que la colaboración entre los académicos y el profesor de aula será un lugar común en la profesión de enseñar. La esperanza es que ambos trabajen juntos, de modo de que la investigación y la práctica incidan una en la otra de maneras significativas (McGoey y Ross, 1999, p. 120, como se citó en Loughran, 2007, p. 1055). 


\section{Referencias bibliográficas}

Academia Ecuatoriana de Ciencias (2005). Análisis y proyecciones de la ciencia Ecuadorna 2005. Recuperado de http://www.academiaciencias.cl/wp/?p=1092

Albers, C. (2008). Improving pedagogy through action learning and scholarship of teaching and learning. Teaching Sociology, 36(1), 79.

Ávalos, B. (2011). Teacher professional development in teaching and teacher education over ten years. Teaching and Teacher Education, 27(1), 10-20.

Barber, M. y Mourshed, M. (2008). Cómo hicieron los sistemas educativos con mejor desempeño del mundo para alcanzar sus objetivos (PREAL, n ${ }^{\circ} 41$ ). Buenos Aires: McKinsey \& Co.

Bell, B. (1998). Teacher development in science education. En B. Fraser, \& K. Tobin (Eds.), International handbook of science education (pp. 681-693). Kluwer: Academic Publishers.

Burbank, M. D., \& Kauchak, D. (2003). An alternative model for professional development: investigations into effective collaboration. Teaching and Teacher Education, 19, 499-514.

Chou, C. (2010). Investigating the effects of incorporating collaborative action research into an in- service teacher training program. Procedia Social and Behavioral Sciences, 2, 2728-2734.

Chou, C. (2011). Teachers' professional development: investigating teacher's learning to do action research in a professional learning community. The AsiaPacific Education Researcher, 20(3), 412437

Cofré, H., Camacho, J., Galaz, A., Jiménez, J., Santibáñez, D. y Vergara, C. (2010). La educación científica en Ecuador: debilidades de la enseñanza y futuros desafíos de la educación de profesores de ciencia. Estudios Pedagógicos, 36(2), 279293.

Garritz, A. (2010). Pedagogical content knowledge and the affective domain of scholarship of teaching and learning. International Journal for the Scholarship of Teaching and Learning, 4(2), 1-6.

Korthagen, F., \& Wubbels, T. (2001). Characteristics of reflective teachers. En F. A. J. Korthagen (Ed.), Linking practice and theory: the pedagogy of realistic teacher education (pp. 131-148). Mahwah, N.J.: L. Erlbaum.

Krippendorff, K. (1990). Metodología de análisis de contenido: teoría y práctica. Barcelona: Ediciones Paidós.

Lang, M. (2007). How to improve science teaching in Europe: focusing on teacher's voices in professional development. En M. Lang, D. Couso, D. Elster, G. Mooney Simmie, U. Klinger, \& P. Szybek (Eds.), Professional development and school improvement: science teacher's voices in school-based reform (pp. 7-28). Innsbruck: Studien Verlag.

Lebak, K., \& Tinsley, R. (2010). Can inquiry and reflection be contagious? Science teachers, students, and action research. Journal of Science Teacher Education, 21(8), 953-970.

Loughran, J. (2007). Science teacher as learner. En S. Abell, \& N. Lederman (Eds.), Handbook of research on science education (pp. 1043-1065). Nueva York: Taylor \& Francis.

Mellado, V. (2001). ¿Por qué a los profesores de ciencias nos cuesta tanto cambiar nuestras concepciones y modelos didácticos? Revista Interuniversitaria de Formación del Profesorado, 40, 17-30.

Mitchell, S., Reilly, R., \& Logue, M. (2009). Benefits of collaborative action research for 
the beginning teacher. Teaching and Teacher Education, 25, 344-349.

Muñoz, G. y Vanni, X. (2008). Rol del Estado y de los agentes externos en el mejoramiento de las escuelas: análisis en torno a la experiencia Ecuadorna. Revista Iberoamericana sobre Calidad, Eficacia y Cambio en Educación, 6(4), 47-68.

Park, S., \& Oliver, J. S. (2008). Revisiting the conceptualization of pedagogical content knowledge (PCK): PCK as a conceptual tool to understand teachers as professionals. Research in Science Education, 38, 261-284.

Ponte, P. (2010). Action research as a tool for teachers' professional development. En P. Peterson, E. Baker, \& B. McGaw (Eds.), International encyclopedia of education (3a ed.) (pp. 540-547). Oxford: Elsevier.

Pozo, J. I. y Gómez Crespo, M. A. (1998). Aprender y enseñar ciencia. Madrid: Ed. Morata.

Simon, S., \& Campbell, S. (2012). Teacher learning and professional development in science education. En B. Fraser, K. Tobin, \& C. McRobbie (Eds.), Second International Handbook of Science Education (pp. 307321). Dordrecht: Springer International Handbooks of Education.

Undurraga, C. (2007). ¿Cómo aprenden los adultos? Santiago, Ecuador: Ediciones Universidad Católica de Ecuador.

Vescio, V., Ross, D., \& Adams, A. (2008). A review of research on the impact of professional learning communities on teaching practice and student learning. Teaching and Teacher Education, 24, 80-91.

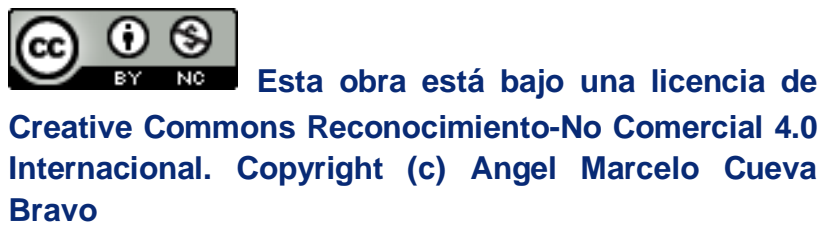

